JIRSS (2020)

Vol. 19, No. 01, pp 21-37

DOI:10.29252/jirss.19.1.21

\title{
Jackknifed Liu-type Estimator in Poisson Regression Model
}

\author{
Ahmed Naziyah Alkhateeb ${ }^{1}$, and Zakariya Yahya Algamal ${ }^{2}$ \\ ${ }^{1}$ Department of Operation Research and Intelligent Techniques, University of Mosul, Mosul, \\ Iraq. \\ ${ }^{2}$ Department of Statistics and Informatics, College of Computer science and Mathematics, \\ University of Mosul, Mosul, Iraq.
}

Received: 24/07/2019, Revision received: 28/04/2020, Published online: 05/05/2020

\begin{abstract}
The Liu estimator has consistently been demonstrated to be an attractive shrinkage method for reducing the effects of multicollinearity. The Poisson regression model is a well-known model in applications when the response variable consists of count data. However, it is known that multicollinearity negatively affects the variance of the maximum likelihood estimator (MLE) of the Poisson regression coefficients. To address this problem, a Poisson Liu estimator has been proposed by numerous researchers. In this paper, a Jackknifed Liu-type Poisson estimator (JPLTE) is proposed and derived. The idea behind the JPLTE is to decrease the shrinkage parameter and, therefore, improve the resultant estimator by reducing the amount of bias. Our Monte Carlo simulation results suggest that the JPLTE estimator can bring significant improvements relative to other existing estimators. In addition, the results of a real application demonstrate that the JPLTE estimator outperforms both the Poisson Liu estimator and the maximum likelihood estimator in terms of predictive performance.
\end{abstract}

Keywords. Multicollinearity, Liu Estimator, Poisson Regression Model, Shrinkage, Monte Carlo Simulation.

Ahmed Naziyah Alkhateeb (ahmed.alkhateeb@uomosul.edu.iq)

Corresponding Author: Zakariya Yahya Algamal (zakariya.algamal@uomosul.edu.iq) 
MSC: 62J12, 62J05.

\section{Introduction}

The Poisson regression model is widely applied in the study of real data problems, such as mortality studies, where the aim is to investigate the number of deaths and health insurance, where the target is to explain the number of claims made by individual Algamal (2012), Algamal (2018), Cameron and Trivedi (2013) and De Jong and Heller (2008).

In dealing with a Poisson regression model, it is assumed that there is no correlation among the explanatory variables. In practice, however, this assumption often does not hold, which causes a problem of multicollinearity. In the presence of multicollinearity, when estimating the regression coefficients for the Poisson regression model using the maximum likelihood (ML) method, the estimated coefficients usually become unstable with a high variance, and therefore low statistical significance Liu (2003). Numerous remedial methods have been proposed to overcome the problem of multicollinearity. The ridge regression method Hoerl and Kennard (1970) has consistently been demonstrated to be an attractive alternative to the ML estimation method.

Kibria (2003)proposed the Liu estimator, which also has the advantages of being a linear function of the shrinkage parameter. This estimator has the advantages of a ridge estimator and also those of a Stein estimator. Mansson et al. (2012) also showed the superiority of the Liu-type estimator, which is a two-parameter estimator, over ridge regression; the Liu-type estimator uses the advantages of both a ridge estimator and a Liu estimator. In a Liu-type estimator, one can use a large shrinkage value, because there is another parameter to make the estimator give a good fit. Although the Liu-type estimator the valuable characteristics, it has a smaller bias. It is possible to reduce the bias by applying a jackknife procedure to a biased estimator. The Jackknife procedure enables experimental data to be processed to produce a statistical estimator for unknown parameters. A truncated sample is used to calculate a specific function of the estimators. The advantage of the jackknife procedure is that it produces an estimator that has a small bias while still using the beneficial properties of large samples Turkan and Ozel (2017).

In this paper, an extension of the work of Akdeniz Duran and Akdeniz (2012) and Yildiz (2018) is proposed and applied to the Liu-type Poisson estimator. The idea behind our proposed estimator is to decrease the shrinkage parameter giving, therefore, 
a resultant estimator with a small amount of bias.

\section{Poisson Regression Model}

The most popular distribution when analyzing count data is the Poisson regression. Data of this type are used in economics, social sciences, and medicine. We know that the form of the Poisson distribution is

$$
f\left(y_{i}\right)=e^{-\mu_{i}} \frac{\mu_{i}^{y_{i}}}{y_{i} !}, \quad y_{i}=0,1,2, \ldots, i=1,2, \ldots, n
$$

Where $y_{i}$ is the response variable, the expected value of a Poisson regression is equal to the exponential distribution, such as

$$
\mu_{i}=e^{x_{i}^{\prime} \beta} .
$$

Here $x_{i}$ is the $\mathrm{i}$-th row of the independent variable $X$ which is $n \times p$ with $p$ variables and $\beta$ is a $1 \times p$ of coefficients. We use the maximum likelihood method to estimate the coefficients of the Poisson regression model, which is considered to be a non-linear model, as follows

$$
\prod_{i=1}^{n} f\left(y_{i}\right)=\prod_{i=1}^{n}\left(e^{-\mu_{i}} \frac{\mu_{i} y_{i}}{y_{i} !}\right) .
$$

The log-likelihood function of (2.3) is

$$
\begin{aligned}
\ell(\beta) & =\sum_{i=1}^{n}\left(y_{i} x_{i}^{\prime} \beta-\exp \left(x_{i}^{\prime} \beta\right)-\ln \left(y_{i} !\right)\right) \\
& =\sum y_{i} x_{i}^{\prime} \beta-\sum \exp \left(x_{i}^{\prime} \beta\right)-\sum \ln \left(y_{i} !\right) .
\end{aligned}
$$

We use the maximum likelihood (ML) method to solve the following equation

$$
\frac{\partial \ell(\beta)}{\partial \beta}=\sum_{i=1}^{n}\left(y_{i}-\exp \left(x_{i}^{\prime} \beta\right)\right) x_{i} .
$$

Since equation (2.5) is non-linear in $\beta$, then by using weighted least square algorithm, we have

$$
\hat{\beta}_{P R}=\left(X^{\prime} \hat{W} X\right)^{-1} X^{\prime} \hat{W} \hat{s},
$$

where $\hat{W}=\operatorname{diag}\left(\hat{\mu}_{i}\right)$ and the i-th element of the row vector $\hat{s}$ is equal to $\log \left(\hat{\mu}_{i}\right)+\frac{y_{i}-\hat{\mu}_{i}}{\hat{\mu}_{i}}$. 
The covariance matrix of $\hat{\beta}_{M L}$ is equal to the second derivatives of equation (2.6), by using the ML method, as follows

$$
\operatorname{Cov}\left(\hat{\beta}_{M L}\right)=\left(X^{\prime} \hat{W} X\right)^{-1}
$$

By tacking the trace of (2.7) we have the mean square error (MSE), as follows

$$
\operatorname{MSE}\left(\hat{\beta}_{M L}\right)=E\left(\hat{\beta}_{M L}-\beta\right)^{\prime}\left(\hat{\beta}_{M L}-\beta\right)=\sum_{j=1}^{p} \frac{1}{\lambda_{j}}
$$

where $\lambda_{j}$ are the eigen-values of $\left(X^{\prime} \hat{W} X\right)^{-1}$. When the independent variables are highly correlated, then the ML estimator is ill-conditioned and this leads to instability and high variance. In this case, it is very hard to interpret the estimated parameters, since the vector of estimated coefficients is, on average, too long.

To avoid this problem one can use the Poisson ridge regression (PRR) proposed by Algamal and Alanaz (2018), Mansson and Shukur (2011) and Singh et al. (1986) by minimizing the weighted sum of squares error (WSSE). Hence $\hat{\beta}_{M L}$ is given by

$$
\begin{aligned}
\hat{\beta}_{P R R} & =\left(X^{\prime} \hat{W} X+k I\right)^{-1} X^{\prime} \hat{W} X \hat{\beta}_{M L} \\
& =\left(X^{\prime} \hat{W} X+k I\right)^{-1} X^{\prime} \hat{W} \hat{s} .
\end{aligned}
$$

The MSE of $\hat{\beta}_{P R R}$ is

$$
\begin{gathered}
\operatorname{MSE}\left(\hat{\beta}_{P R R}\right)=\sum_{j=1}^{J} \frac{\lambda_{j}}{\left(\lambda_{j}+k\right)^{2}}+\beta^{\prime} k^{2}\left(X^{\prime} \hat{W} X+k I\right)^{-2} \beta \\
=\gamma_{1}(k)+\gamma_{2}(k), \\
\therefore M S E\left(\hat{\beta}_{P R R}\right)=\operatorname{VAR}\left(\hat{\beta}_{P R R}\right)+\operatorname{Bias}\left(\hat{\beta}_{P R R}\right),
\end{gathered}
$$

where $\gamma_{1}(k)$ is the variance and $\gamma_{2}(k)$ is the bias part of $\hat{\beta}_{P R R}$.

The MSE of $\hat{\beta}_{P R R}$ is lower than the estimate $\hat{\beta}_{M L}$ when we find $k$ (where $k$ may take any value between zero and infinity) such that the reduction in the variance part is greater than the increase in the squared part, for this reason the PRR estimation is better than ML estimation. Furthermore, the PRR is a simple method since it does not require any changes to the Poisson regression. 


\section{Liu-type Poisson Regression Estimator}

The most popular biased estimator is Karbalaee et al. (2019) which adopts the Poisson regression model and is defined as follows Algamal (2018), Arashi et al. (2014) and Arashi et al. (2017)

$$
\hat{\beta}_{P L E}=\left(X^{\prime} \hat{W} X+I\right)^{-1}\left(X^{\prime} \hat{W} X+d I\right) \hat{\beta}_{M L} .
$$

Where $0<d<1$, where the MSE of $\hat{\beta}_{P L E}$ is lower than MSE of $\hat{\beta}_{M L}$ Liu (2003) which is equal to

$$
\hat{\beta}_{P L E}=\sum_{j=1}^{J} \frac{\left(\lambda_{j}+d\right)^{2}}{\lambda_{j}\left(\lambda_{j}+I\right)^{2}}+(d-I)^{2} \sum_{j=1}^{J} \frac{\alpha_{j}^{2}}{\left(\lambda_{j}+I\right)^{2}},
$$

where $\alpha_{j}^{2}$ is defined as the $\mathrm{j}$-th element of $\gamma \beta$, where $\gamma$ is the eigenvector defined by $X^{\prime} \hat{W} X=\gamma^{\prime} \Lambda \gamma$ and $\Lambda$ is the diagonal matrix with elements equal to $\lambda_{j}$.

For the estimator $\hat{\beta}_{P L E}$ the matrix of cross-products used by Kibria (2003) is replaced with the weighted matrix of cross-products and the ordinary least square estimator of $\beta$ is replaced with the ML estimator Hinkley (1977). The MSE of $\hat{\beta}_{P L E}$ is

$$
\begin{aligned}
\operatorname{MSE}\left(\hat{\beta}_{P L E}\right) & =E\left(\hat{\beta}_{P L E}-\beta\right)^{\prime}\left(\hat{\beta}_{P L E}-\beta\right) \\
& =\operatorname{tr}\left[\left(\hat{\beta}_{M L}-\beta\right)^{\prime}\left(\hat{\beta}_{M L}-\beta\right) S^{\prime} S\right]+\beta^{\prime} k^{2}\left(X^{\prime} \hat{W} X+k I\right)^{-2} \beta .
\end{aligned}
$$

By taking the trace for the equation above, we have

$$
\begin{gathered}
\operatorname{MSE}\left(\hat{\beta}_{P L E}\right)=\sum_{j=1}^{J} \frac{\left(\lambda_{j}+d\right)^{2}}{\lambda_{j}\left(\lambda_{j}+I\right)^{2}}+(d-I)^{2} \sum_{j=1}^{J} \frac{\alpha_{j}^{2}}{\left(\lambda_{j}+I\right)^{2}}, \\
\operatorname{MSE}\left(\hat{\beta}_{P L E}\right)=\omega(d)_{1}+\omega(d)_{2} .
\end{gathered}
$$

From equation (3.3), the MSE of $\left(\hat{\beta}_{P L E}\right)$ is equal to $\omega(d)_{1}$, which is the variance, and the biased part, which is represented by $\omega(d)_{2}$.

To show that $M S E\left(\hat{\beta}_{P L E}\right)<M S E\left(\beta_{M L}\right)$ we take the first derivative of equation (3.3) with respect to $d$ as follows Algamal (2018)

$$
\frac{\partial\left(M S E\left(\hat{\beta}_{P L E}\right)\right)}{\partial(d)}=2 \sum_{j=1}^{J} \frac{\lambda_{j}+d}{\lambda_{j}\left(\lambda_{j}+I\right)^{2}}+2(d-I) \sum_{j=1}^{J} \frac{\alpha_{j}^{2}}{\left(\lambda_{j}+I\right)^{2}} .
$$


Since $0<d<1$, by inserting $d=1$ into equation (3.4), we have

$$
\begin{aligned}
\frac{\partial\left(\operatorname{MSE}\left(\hat{\beta}_{P L E}\right)\right)}{\partial(d)} & =2 \sum_{j=1}^{J} \frac{\lambda_{j}+1}{\lambda_{j}\left(\lambda_{j}+I\right)^{2}} \\
& =2 \sum_{j=1}^{J} \frac{1}{\lambda_{j}\left(\lambda_{j}+I\right)} ; \lambda_{j}>0 .
\end{aligned}
$$

The optimal value of $d_{j}$ can be found by setting equation (3.4) to zero and solving for $d_{j}$, which may then be shown as

$$
d_{j}=\frac{\alpha_{j}^{2}-1}{\frac{1}{\lambda_{j}+\alpha_{j}^{2}}} .
$$

Liu upgraded this by proposing the Liu-type estimator to overcome the problem of severe multicollinearity. The Liu-type estimator is defined as follows Algamal (2018)

$$
\hat{\beta}_{P L T E}=\left(X^{\prime} \hat{W} X+k I\right)^{-1}\left(X^{\prime} \hat{W} X-d I\right) \hat{\beta}_{M L},
$$

where $-\infty<d<\infty$ and $k \geq 0$. The Liu-type estimator is superior to the ridge estimator Liu (2003). Liu noted that when there is sever multicollinearity, the shrinkage ridge parameter may not fully address the ill-conditioning problem. He therefore modified Liu estimator and suggested Liu-type estimator. The MSE of $\hat{\beta}_{P L T E}$ is

$$
\operatorname{MSE}\left(\hat{\beta}_{\text {PLTE }}\right)=\sum_{j=1}^{J} \frac{\left(\lambda_{j}-d\right)^{2}}{\lambda_{j}\left(\lambda_{j}+k\right)^{2}}+(d+k)^{2} \sum_{j=1}^{J} \frac{\alpha_{j}^{2}}{\left(\lambda_{j}+k\right)^{2}} .
$$

\section{The Proposed Estimator: Jackknifed Liu-type Estimator}

In this section, the new estimator is introduced and derived. Let $M=\left(m_{1}, m_{2}, \ldots, m_{p}\right)$ and $\Lambda=\operatorname{diag}\left(\lambda_{1}, \lambda_{2}, \ldots, \lambda_{p}\right)$, respectively, be the matrices of the eigenvectors and eigenvalues of a symmetric matrix $C=X^{\prime} W X$ that has an eigenvalues and eigenvectors decomposition of the form $C=T \Lambda T^{\prime}$, where $T$ is an orthogonal matrix and $\Lambda$ is a diagonal matrix. Consequently, the Poisson regression estimator of equation (2.6), $\hat{\beta}_{P R}$, can be written as

$$
\begin{aligned}
& \hat{\gamma} P R=\Lambda^{-1} S^{\prime} \hat{W} \hat{v} \\
& \hat{\beta}_{P R}=M \hat{\gamma}_{P R} .
\end{aligned}
$$


In Liu (2003), a new estimator is proposed for $\gamma$. This estimator is biased and is called Liu-type estimator (LTE), and is defined as follows

$$
\begin{aligned}
\hat{\gamma} \operatorname{PLTE}(k, d) & =(\Lambda+k I)^{-1}\left(M^{\prime} y-d \hat{\gamma} P R\right) \\
& =(\Lambda+k I)^{-1}\left(M^{\prime} y-d \Lambda^{-1} M^{\prime} y\right) \\
& =\left[I-(\Lambda+k I)^{-1}(k+d)\right] \hat{\gamma} P R \\
& =H(k, d) \hat{\gamma} P R
\end{aligned}
$$

where

$$
H(k, d)=(\Lambda+k I)^{-1}(\Lambda-d I),
$$

$\hat{\gamma}_{P L T E}$ has a bias vector defined as

$$
\operatorname{bias}(\hat{\gamma} P L T E)=(H(k, d)-I) \gamma,
$$

and a covariance matrix

$$
\operatorname{cov}\left(\hat{\gamma}_{P L T E}\right)=H(k, d) \Lambda^{-1} H(k, d) \text {. }
$$

By using Hinkley (1977), Singh et al. (1986), Nyquist (1988) and Batah et al. (2008) we can propose a jackknifed form of $\hat{\gamma}$ PLTE. In Quenouille (1956) and Tukey (1958) the jackknife method was introduced to reduce the value of the bias. In Hinkley (1977) it was stated that, with a few exceptions, the jackknife technique can be applied to balanced models. The jackknifed estimator, after some algebraic manipulations, is obtained by deleting the i-th observation $\left(m_{i}^{\prime}, y_{i}\right)$

$$
\begin{aligned}
\hat{\gamma} \operatorname{PLTE}(k, d) & =\left(M_{-i}^{\prime} \hat{W}_{-i} M_{-i}+k I\right)^{-1}\left(M_{-i}^{\prime} \hat{W}_{-i} M_{-i}-d I\right)\left(M_{-i}^{\prime} \hat{W}_{-i} M_{-i}\right)^{-1} M_{-i}^{\prime} y_{-i} \\
& =\left(A-M_{-i}^{\prime} \hat{W}_{-i} M_{-i}\right)^{-1}\left(M^{\prime} y-m_{i} y_{i}\right) \\
& =\left(A^{-1}+\frac{A^{-1} m_{i} w_{i} m_{i}^{\prime} A^{-1}}{1-m_{i}^{\prime} A^{-1} m_{i}}\right) \\
& =A^{-1} M^{\prime} y-A^{-1} m_{i} y_{i}\left(\frac{A^{-1} m_{i} w_{i} m_{i}^{\prime} A^{-1}}{1-m_{i}^{\prime} A^{-1} m_{i}} M^{\prime} y-\frac{A^{-1} m_{i} w_{i} m_{i}^{\prime} A^{-1}}{1-m_{i}^{\prime} A^{-1} m_{i}} m_{i} y_{i}\right) \\
& =\hat{\gamma} \operatorname{PLTE}(k, d)+A^{-1} m_{i} y_{i}\left(1+\frac{m_{i}^{\prime} A^{-1} m_{i}}{1-m_{i}^{\prime} A^{-1} m_{i}}\right)+\frac{A^{-1} m_{i} w_{i} m_{i}^{\prime}}{1-m_{i}^{\prime} A^{-1} m_{i}} \hat{\gamma}_{\operatorname{PLTE}}(k, d) \\
& =\hat{\gamma} \operatorname{PLTE}(k, d)-A^{-1} m_{i} \frac{A^{-1} m_{i}\left(y_{i}-m_{i}^{\prime} \hat{\gamma}_{P L T E}(k, d)\right)}{1-m_{i}^{\prime} A^{-1} m_{i}} \\
& =\hat{\gamma} \operatorname{PLTE}(k, d)-\frac{A^{-1} m_{i} e_{i}}{1-f_{i}},
\end{aligned}
$$

where $m_{i}^{\prime}$ is the $\mathrm{i}$-th row of the matrix $M, e_{i}=y_{i}-m_{i}^{\prime} \hat{\gamma}_{P L T E}(k, d)$ is the Liu-type residual, $M_{-i}^{\prime} \hat{W}_{-i} M_{-i}=M^{\prime} \hat{W} M-m_{i} \hat{w}_{i} m_{i}^{\prime}, M_{-i}^{\prime} y_{-i}=M^{\prime} y-m_{i} y_{i}$ and $f_{i}=m_{i}^{\prime} A^{-1} m_{i}$ is the distance factor and $A^{-1}=(\Lambda+k I)^{-1}\left(I-d \Lambda^{-1}\right)=H(k, d) \Lambda^{-1}$. 
The bias part and the variance of $\hat{\gamma}_{\operatorname{PLTE}}(k, d)$ are obtained as, respectively

$$
\begin{gathered}
\operatorname{Bias}(\hat{\gamma} \text { PLTE }(k, d))=-(I-H(k, d))^{2} \gamma, \\
\operatorname{cov}(\hat{\gamma} \text { PLTE }(k, d))=\sigma^{2}\left((2 I-H(k, d)) H(k, d) \Lambda^{-1} H(k, d)^{\prime}(2 I-H(k, d))^{\prime} .\right.
\end{gathered}
$$

The MSEMs of the jackknifed Poisson Liu-type estimator (JPLTE) and the Poisson Liu-type estimator (PLTE) are given as follows

$$
\begin{aligned}
\operatorname{MSEM}\left(\hat{\gamma}_{\text {JPLTE }}(k, d)\right) & =\operatorname{cov}\left(\hat{\gamma}_{\text {JPLTE }}(k, d)\right)+\operatorname{Bias}\left(\hat{\gamma}_{\text {JPLTE }}(k, d)\right) \operatorname{Bias}\left(\hat{\gamma}_{\text {JPLTE }}(k, d)\right)^{\prime} \\
& =(2 I-H(k, d)) H(k, d) \Lambda^{-1} H, \\
\operatorname{MSEM}\left(\hat{\gamma}_{J P L T E}\right)= & H(k, d) \Lambda^{-1} H(k, d)^{\prime}+(H(k, d)-I) \gamma \gamma^{\prime}(H(k, d)-I) .
\end{aligned}
$$

Let

$$
\begin{aligned}
\Delta_{1} & =\operatorname{MSEM}\left(\hat{\gamma}_{J L T E}(k, d)\right)-\operatorname{MSEM}\left(\hat{\gamma}_{M J L T E}(k, d)\right) \\
& =\sum+H^{*}(k, d)^{2} \gamma \gamma^{\prime} H^{*}(k, d)^{2^{\prime}}-H^{*}(k, d) W \gamma \gamma^{\prime} W^{\prime} H^{*}(k, d)^{\prime},
\end{aligned}
$$

where $\sum$ is symmetric and positive definite matrix. Therefore, the difference $\Delta_{1}$ is nonnegative definite, if and only if $L^{-1} \Delta_{1} L^{-1^{\prime}}$ is nonnegative definite. Also, the matrix $L^{-1} \Delta_{1} L^{-1^{\prime}}$ can be written as

$$
L^{-1} \Delta_{1} L^{-1^{\prime}}=L^{-1}\left(\sum+H^{*}(k, d)^{2} \gamma \gamma^{\prime} H^{*}(k, d)^{2^{\prime}}\right) L^{-1^{\prime}}-\gamma \gamma^{\prime},
$$

the difference $\Delta_{1}$ is a nonnegative definite matrix. If and only if $L^{-1} \Delta_{1} L^{-1^{\prime}}$ is a nonnegative definite matrix. Since the matrix $\left(\sum+H^{*}(k, d)^{2} \gamma \gamma^{\prime} H^{*}(k, d)^{2^{\prime}}\right)$ is symmetric and positive definite, we may conclude that $L^{-1} \Delta_{1} L^{-1^{\prime}}$ is a nonnegative definite, if and only if the inequalityYıldız (2018) holds

$$
\gamma^{\prime}\left[L^{-1}\left(\sum+H^{*}(k, d)^{2} \gamma \gamma^{\prime} H^{*}(k, d)^{2^{\prime}}\right) L^{-1^{\prime}}\right]^{-1} \gamma \leq 1
$$

\section{Simulation Results}

In this section, a Monte Carlo simulation experiment is conducted to examine the performance of the new estimator with different degrees of multicollinearity. The response variable of $\mathrm{n}$ observations is generated from the Poisson regression model by

$$
\theta_{i}=\exp \left(x_{i}^{\prime} \beta\right)
$$


where $\beta=\left(\beta_{0}, \beta_{1}, \ldots, \beta_{p}\right)$ with $\sum_{j=1}^{p} \beta_{j}^{2}=1$ and $\beta_{1}=\beta_{2}=\ldots=\beta_{p}$ Kibria et al. (2015) and Kibria (2003). In addition, because the value of intercept, $\beta_{0}$, has an effect on $\theta_{i}$, three values are chosen for $\beta_{0} \in\{1,0,-1\}$, where decreasing the value of $\beta_{0}$ leads to a lower average value of $\theta_{i}$, which leads to less variation Arashi et al. (2018), Asar and Genç (2017) and Mansson et al. (2012).

The explanatory variables $x_{i}^{\prime}=\left(x_{i 1}, x_{i 2}, \ldots, x_{i n}\right)$ have been generated from the following formula

$$
x_{i j}=\left(1-\rho^{2}\right)^{1 / 2} w_{i j}+\rho w_{i p} \quad, \quad \mathrm{i}=1,2, \ldots, \mathrm{n} \quad, \quad \mathrm{j}=1,2, \ldots, \mathrm{p},
$$

where $\rho$ represents the correlation between the explanatory variables and the $w_{i j}$ 's are independent standard normal pseudo-random numbers. Because the sample size has a direct impact on the prediction accuracy, three representative values of sample size are considered: 30,50 , and 100 . In addition, the number of the explanatory variables is considered to be $p=4$ and $p=8$ because increasing the number of explanatory variables can lead to an increase in the MSE. Further, because we are interested in the effect of multicollinearity, in which the degrees of correlation are considered to be important, three values of the pairwise correlation are considered with $\rho=\{0.90,0.95,0.99\}$. For a combination of these different values of $n, p, \beta$, and $\rho$ the generated data are repeated 1000 times and the estimated averaged mean squared error (EMSE) is calculated as

$$
\operatorname{EMSE}(\hat{\beta})=\frac{1}{1000} \sum_{i=1}^{1000}(\hat{\beta}-\beta)^{\prime}(\hat{\beta}-\beta),
$$

where $\hat{\beta}$ is the estimated coefficient for the estimator used. For the value of $k$, the best method of Kibria et al. (2015) was used as

$$
\mathrm{k}=\max \left\{\frac{1}{m_{j}}\right\}, j=1,2, \ldots, p,
$$

where $m_{j}=\sqrt{\hat{\sigma}^{2} / \hat{\alpha}_{j}^{2}}$.

As is used in Yildiz (2018) and Akdeniz Duran and Akdeniz (2012), the value of $d$ is set as 0.5 and 0.75 . For the value of $k$, it can be computed as in Hoerl and Kennard (1970).

The estimated MSEs of Eq. (5.3) for MLE, PLE, PLTE, and JPLTE, for all the combination of $n, p, \beta_{0}$, and $\rho$, are summarized in Tables 1-3. Several observations can be made. 
Table 1: MSE values when $\beta_{0}=-1$

\begin{tabular}{|c|c|c|c|c|c|c|c|c|c|}
\hline & & & \multicolumn{3}{|c|}{$\mathrm{d}=0.5$} & \multicolumn{3}{|c|}{$\mathrm{d}=0.75$} & \multirow[b]{2}{*}{ JPLTE } \\
\hline & & & ML & PLE & PLTE & JPLTE & PLE & PLTE & \\
\hline$p$ & $n$ & $\rho$ & & & & & & & \\
\hline \multirow[t]{9}{*}{4} & 30 & 0.90 & 5.037 & 1.076 & 0.923 & 0.615 & 1.101 & 0.948 & 0.649 \\
\hline & & 0.95 & 5.665 & 1.307 & 1.156 & 1.064 & 1.331 & 1.18 & 1.088 \\
\hline & & 0.99 & 6.063 & 1.957 & 1.805 & 1.596 & 1.981 & 1.829 & 1.62 \\
\hline & 50 & 0.90 & 3.408 & 0.709 & 0.556 & 0.526 & 0.733 & 0.58 & 0.55 \\
\hline & & 0.95 & 4.483 & 0.981 & 0.828 & 0.635 & 1.005 & 0.852 & 0.659 \\
\hline & & 0.99 & 4.675 & 1.298 & 1.145 & 1.525 & 1.322 & 1.169 & 1.549 \\
\hline & 100 & 0.90 & 3.251 & 0.511 & 0.358 & 0.497 & 0.535 & 0.382 & 0.521 \\
\hline & & 0.95 & 3.461 & 0.635 & 0.482 & 0.524 & 0.659 & 0.506 & 0.548 \\
\hline & & 0.99 & 4.216 & 1.661 & 1.508 & 1.3 & 1.685 & 1.532 & 1.324 \\
\hline \multirow[t]{9}{*}{8} & 30 & 0.90 & 5.142 & 1.278 & 1.125 & 0.807 & 1.302 & 1.149 & 0.831 \\
\hline & & 0.95 & 5.761 & 1.509 & 1.356 & 1.256 & 1.533 & 1.38 & 1.28 \\
\hline & & 0.99 & 6.176 & 2.159 & 2.006 & 1.788 & 2.183 & 2.03 & 1.812 \\
\hline & 50 & 0.90 & 3.677 & 0.911 & 0.758 & 0.718 & 0.935 & 0.782 & 0.742 \\
\hline & & 0.95 & 4.82 & 1.183 & 1.03 & 0.827 & 1.207 & 1.054 & 0.851 \\
\hline & & 0.99 & 5.145 & 1.5 & 1.347 & 1.717 & 1.524 & 1.371 & 1.741 \\
\hline & 100 & 0.90 & 3.587 & 0.703 & 0.55 & 0.689 & 0.727 & 0.574 & 0.713 \\
\hline & & 0.95 & 3.862 & 0.827 & 0.675 & 0.716 & 0.851 & 0.699 & 0.74 \\
\hline & & 0.99 & 4.42 & 1.853 & 1.7 & 1.492 & 1.877 & 1.724 & 1.516 \\
\hline
\end{tabular}

First, in terms of $\rho$ values, there is an increase in the MSE value when the correlation degree increases, regardless of the values $n, p$ and $\beta_{0}$. However, JPLTE performs better than PLTE, PLE, and MLE for all cases. For instance, in Table 1, when $p=4, n=100$, and $\rho=0.95$, the MSE of JPLTE is about $51.34 \%, 32.74 \%$, and $18.31 \%$ lower than the MSEs of MLE, PLE, and PLTE, respectively.

Second, regarding the number of explanatory variables, it is easily seen that there is an increase in the MSE values when $p$ increase from four variables to eight variables. Although this increase can affect the quality of an estimator, JPLTE achieves the lowest MSE when compare with the other estimators used, for different $n, \rho$ and $\beta_{0}$. Third, concerning the value of $n$, the MSE values decrease when $n$ increases, regardless of the values of $\rho, p$ and $\beta_{0}$. However, JPLTE still consistently outperforms the others by providing the lowest MSE. 
Table 2: MSE values when $\beta_{0}=0$

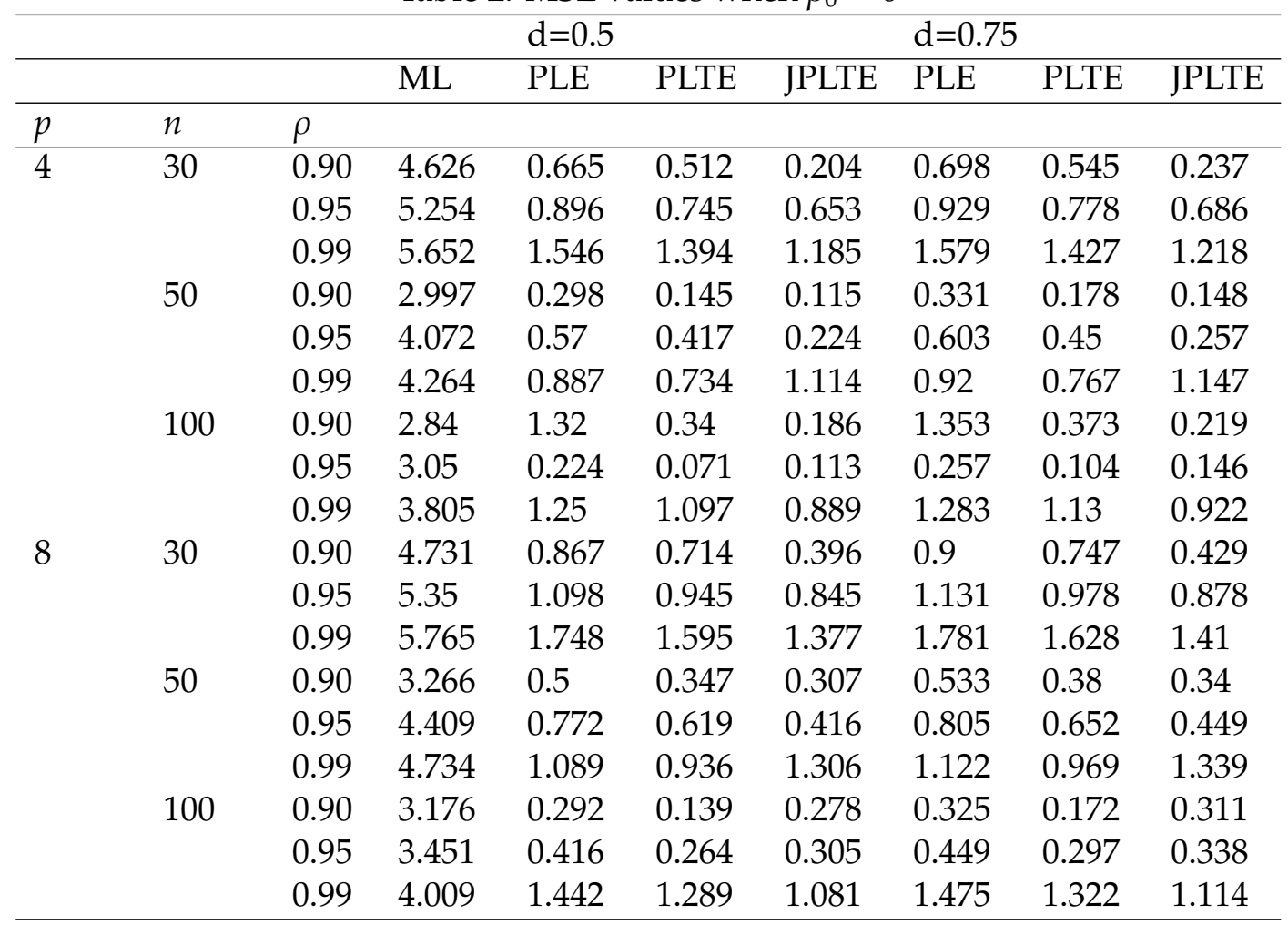

Fourth, in terms of the value of the intercept and for given values of $\rho, p$, and $n$, JPLTE always shows a smaller MSE than andthe other estimators.

To summarize, for all the values of $n, \rho, p$ and $\beta_{0}$ considered, the proposed estimator, JPLTE, is superior to PLTE and PLT, clearly indicating that the new proposed estimator is more efficient.

\section{Real Application}

To investigate the usefulness of our new estimator, we apply the proposed estimator to the Spanish La Liga, football season 2016-2017. There are data here for 20 teams. The response variable represents the number of matches won. The six explanatory variables considerable are the number of yellow cards $\left(x_{1}\right)$, the number of red cards 
$\left(x_{2}\right)$, the total number of substitutions $\left(x_{3}\right)$, the number of matches with 2.5 goals on average $\left(x_{4}\right)$, the number of matches that ended with goals $\left(x_{5}\right)$, and the ratio of the goals scoreds to the number of matches $\left(x_{6}\right)$.

Table 3: MSE values when $\beta_{0}=1$

\begin{tabular}{|c|c|c|c|c|c|c|c|c|c|}
\hline & & & & $\mathrm{d}=0.5$ & & & $\mathrm{~d}=0.7$ & & \\
\hline & & & ML & PLE & PLTE & JPLTE & PLE & PLTE & JPLTE \\
\hline$p$ & $n$ & $\rho$ & & & & & & & \\
\hline 4 & 30 & 0.90 & 5.229 & 1.268 & 1.115 & 0.807 & 1.281 & 1.148 & 0.84 \\
\hline & & 0.95 & 5.857 & 1.499 & 1.348 & 1.256 & 1.512 & 1.381 & 1.289 \\
\hline & & 0.99 & 6.255 & 2.149 & 1.997 & 1.788 & 2.162 & 2.03 & 1.821 \\
\hline & 50 & 0.90 & 3.6 & 0.901 & 0.748 & 0.718 & 0.914 & 0.781 & 0.751 \\
\hline & & 0.95 & 4.675 & 1.173 & 1.02 & 0.827 & 1.186 & 1.053 & 0.86 \\
\hline & & 0.99 & 4.867 & 1.49 & 1.337 & 1.717 & 1.503 & 1.37 & 1.75 \\
\hline & 100 & 0.90 & 3.443 & 1.923 & 0.943 & 0.789 & 1.936 & 0.976 & 0.822 \\
\hline & & 0.95 & 3.653 & 0.827 & 0.674 & 0.716 & 0.84 & 0.707 & 0.749 \\
\hline & & 0.99 & 4.408 & 1.853 & 1.7 & 1.492 & 1.866 & 1.733 & 1.525 \\
\hline 8 & 30 & 0.90 & 5.334 & 1.47 & 1.317 & 0.999 & 1.483 & 1.35 & 1.032 \\
\hline & & 0.95 & 5.953 & 1.701 & 1.548 & 1.448 & 1.714 & 1.581 & 1.481 \\
\hline & & 0.99 & 6.368 & 2.351 & 2.198 & 1.98 & 2.364 & 2.231 & 2.013 \\
\hline & 50 & 0.90 & 3.869 & 1.103 & 0.95 & 0.91 & 1.116 & 0.983 & 0.943 \\
\hline & & 0.95 & 5.012 & 1.375 & 1.222 & 1.019 & 1.388 & 1.255 & 1.052 \\
\hline & & 0.99 & 5.337 & 1.692 & 1.539 & 1.909 & 1.705 & 1.572 & 1.942 \\
\hline & 100 & 0.90 & 3.779 & 0.895 & 0.742 & 0.881 & 0.908 & 0.775 & 0.914 \\
\hline & & 0.95 & 4.054 & 1.019 & 0.867 & 0.908 & 1.032 & 0.9 & 0.941 \\
\hline & & 0.99 & 4.612 & 2.045 & 1.892 & 1.684 & 2.058 & 1.925 & 1.717 \\
\hline
\end{tabular}

First, the deviance test Montgomery et al. (2015), Karbalaee et al. (2019) and Liu (1993) is used to check whether the Poisson regression model fits these data well or not. The result of the residual deviance test is equal to 7.394 with 14 degrees of freedom and the $\mathrm{p}$-value is 0.916 . This result indicates that the Poisson regression model fits these data very well.

Second, to check whether or not there are relationships between the explanatory variables, Figure 1 displays the correlation matrix among the six explanatory variables. It is obvious that there are correlations greater than 0.82 between $x_{1}$ and $x_{6}, x_{1}$ and $x_{4}$, 
$x_{2}$ and $x_{4}$, and, $x_{4}$ and $x_{6}$.

Third, to test the existence of multicollinearity, the eigenvalues of the matrix $X^{\prime} \hat{W} X$ are obtained as 988.628, 142.907, 75.560, 38.999, 21.424, and 1.105. The condition number $\mathrm{CN}=\sqrt{\lambda_{\max } / \lambda_{\min }}$ of the data is determined to be 29.905 indicating that there is a multicollinearity issue.

The estimated Poisson regression coefficients and MSE values for the MLE, PLE, PLTE, and JPLTE estimators when $d=0.75$ are listed in Table 4 . Table 4, clearly shows that the JPLTE estimator shrinks the value of the estimated coefficients efficiently. In terms of MSE, the JPLTE achieves the lowest MSE.

Table 4: The estimated coefficients and MSE values for the MLE, PLE, PLTE, and JPLTE estimators.

\begin{tabular}{lllll}
\hline & MLE & PLE & PLTE & JPLTE \\
\hline$\hat{\beta}_{1}$ & -1.219 & -1.057 & -0.116 & -0.088 \\
$\hat{\beta}_{2}$ & 0.441 & 0.237 & 0.104 & 0.051 \\
$\hat{\beta}_{3}$ & 0.575 & 0.364 & 0.016 & 0.012 \\
$\hat{\beta}_{4}$ & -3.476 & -1.102 & -0.738 & -0.514 \\
$\hat{\beta}_{5}$ & -2.432 & -1.311 & -0.878 & -0.577 \\
$\hat{\beta}_{6}$ & 5.121 & 3.071 & 1.214 & 1.017 \\
& & & & \\
\hline MSE & 3.508 & 1.144 & 1.057 & 0.868
\end{tabular}

\section{Conclusions}

In this paper, a new Poisson Liu-type estimator is proposed to overcome the multicollinearity problem in the Poisson regression model. From the Monte Carlo simulation studies, the new estimator, a jackknifed Liu-type estimator, has a better performance than MLE, PLT, and PLTE, in terms of MSE. Additionally, a real data application is 
considered to illustrate the benefits of using the new estimator in the context of the Poisson regression model. The superiority of the JPLTE based on the resulting MSE is observed and it was shown that the results are consistent with the Monte Carlo simulation results. In conclusion, the use of the new estimator, JPLTE, is recommended when multicollinearity is present in a Poisson regression model.

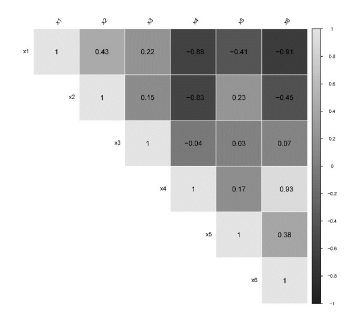

Figure 1: The correlation matrix among the six explanatory variables.

\section{Acknowledgement}

The research is supported by College of Computer Sciences and Mathematics, University of Mosul, Republic of Iraq.

\section{References}

Algamal, Z. Y. (2012), Diagnostic in poisson regression models. Electronic Journal of Applied Statistical Analysis, 5(2), 178-186.

Algamal, Z. Y. (2018), Biased estimators in Poisson regression model in the presence of multicollinearity: A subject review. Al-Qadisiyah Journal for Administrative and Economic Sciences, 20(1), 37-43.

Algamal, Z. Y. (2018), A new method for choosing the biasing parameter in ridge estimator for generalized linear model. Chemometrics and Intelligent Laboratory Systems, Elsevier, 183, 96-101. 
Algamal, Z. Y. and Alanaz, M. M. (2018), Proposed methods in estimating the ridge regression parameter in Poisson regression model. Electronic Journal of Applied Statistical Analysis, 11, 506-515.

Arashi, M., Kibria, B. G., Norouzirad, M. and Nadarajah, S. (2014), Improved preliminary test and Stein-rule Liu estimators for the ill-conditioned elliptical linear regression model. Journal of Multivariate Analysis, Elsevier, 126, 53-74.

Arashi, M., Nadarajah, S. and Akdeniz, F. (2017), The distribution of the Liu-type estimator of the biasing parameter in elliptically contoured models. Communications in Statistics-Theory and Methods, Taylor and Francis, 46, 3829-3837.

Arashi, M., Norouzirad, M., Ahmed, S. E. and Yüzba and scedil, and imath, B. (2018), Rank-based Liu regression. Computational Statistics, Springer, 33, 1525-1561.

Asar, Y., and Genç, A. (2017), A New Two-Parameter Estimator for the Poisson Regression Model. Iranian Journal of Science and Technology, Transactions A: Science, 42(2), 793-803.

Batah, F. S. M., Ramanathan, T. V., and Gore, S. D. (2008), The efficiency of modified jackknife and ridge type regression estimators: a comparison. Surveys in Mathematics and its Applications, 3, $111-122$.

Cameron, A. C., and Trivedi, P. K. (2013), Regression analysis of count data. Cambridge university press, 53.

De Jong, P., and Heller, G. Z. (2008), Generalized linear models for insurance data. Cambridge University Press Cambridge, 10.

Akdeniz Duran, E., and Akdeniz, F. (2012), Efficiency of the modified jackknifed Liutype estimator. Statistical Papers, 53(2), 265-280.

Hinkley, D. V. (1977), Jackknifing in unbalanced situations. Technometrics, 19(3), 285-292.

Hoerl, A. E., and Kennard, R. W. (1970), Ridge regression: Biased estimation for nonorthogonal problems. Technometrics, 12(1), 55-67.

Karbalaee, M. H., Tabatabaey, S. M. M. and Arashi, M. (2019), On the Preliminary Test Generalized Liu Estimator with Series of Stochastic Restrictions. Journal of The Iranian Statistical Society, 18, 113-131. 
Liu, K. (1993), A new class of blased estimate in linear regression. Communications in Statistics-Theory and Methods, 22(2), 393-402.

Kibria, B. M. G. (2003), Performance of some new ridge regression estimators. Communications in Statistics - Simulation and Computation, 32(2), 419-435 .

Kibria, B. G., Mansson, K., and Shukur, G. (2015), A simulation study of some biasing parameters for the ridge type estimation of Poisson regression. Communications in Statistics-Simulation and Computation, 44(4), 943-957.

Liu, K. (2003), Using Liu-type estimator to combat collinearity. Communications in Statistics-Theory and Methods, 32(5), 1009-1020.

Mansson, K., Kibria, B. G., Sjolander, P., and Shukur, G. (2012), Improved Liu estimators for the Poisson regression model. International Journal of Statistics and Probability, 1(1), 2.

Månsson, K., and Shukur, G. (2011), A Poisson ridge regression estimator. Economic Modelling, 28(4), 1475-1481.

Montgomery, D. C., Peck, E. A., and Vining, G. G. (2015), Introduction to linear regression analysis. New York: John Wiley and Sons.

Nyquist, H. (1988), Applications of the jackknife procedure in ridge regression. Computational Statistics and Data Analysis, 6(2), 177-183.

Quenouille, M. H. (1956), Notes on bias in estimation. Biometrika, 43(3/4), 353-360.

Rashad, N. K. and Algamal, Z. Y. (2019), A New Ridge Estimator for the Poisson Regression Model. Iranian Journal of Science and Technology, Transactions A: Science, Springer., 43, 2921-2928.

Singh, B., Chaubey, Y., and Dwivedi, T. (1986), An almost unbiased ridge estimator. Sankhyā: The Indian Journal of Statistics, Series B, 342-346.

Tukey, J. (1958), Bias and confidence in not quite large samples. Annals of Mathematical Statistics, 29(614).

Türkan, S., and Özel, G. (2015), A new modified Jackknifed estimator for the Poisson regression model. Journal of Applied Statistics, 43(10), 1892-1905. 
Türkan, S., and Özel, G. (2017), A Jackknifed estimators for the Negative Binomial regression model. Communications in Statistics-Simulation and Computation, 47(6), $1845-1865$.

Yild1z, N. (2018), On the performance of the Jackknifed Liu-type estimator in linear regression model. Communications in Statistics-Theory and Methods, 47(9), 2278-2290. 